\title{
First report of leaf spot caused by Pseudocercospora puerariicola on kudzu (Pueraria lobata) in Jiangxi China
}

\author{
Ming Feng Yan ${ }^{1} \cdot$ Man Fei Zou ${ }^{1} \cdot$ Bing Liu $^{1} \cdot$ Yuan Xiu Wang ${ }^{1} \cdot$ Gui Hong Xiong ${ }^{1} \cdot$ Jun Xi Jiang ${ }^{1}$ \\ Received: 22 March 2018 / Accepted: 9 July 2018 / Published online: 16 July 2018 \\ (C) Società Italiana di Patologia Vegetale (S.I.Pa.V.) 2018
}

In June 2017, a leaf spot disease on Kudzu (Pueraria lobata) was observed in Guixi city of Jiangxi province, China, with a disease incidence of approximately $30 \%$. The symptoms initially appeared as dark brown lesions surrounded by yellow halos, and later became larger (5-12 $\mathrm{mm}$ in diameter), circular or angular spots with gray centers and dark brown margins. Conidiophores arising from stromata were densely fasciculate, pale olivaceous to pale brown, erect, straight or slightly curved, unbranched, 18.0 to $73.0 \times 2.7$ to $4.3 \mu \mathrm{m}$, and 0 to 3 septate. Conidiogenous loci were inconspicuous, neither thickened nor darkened. Conidia were obclavate, pale olivaceous, straight to slightly curved, with rounded apex and base truncated, 36.4 to $137.8 \times 3.4$ to $5.0 \mu \mathrm{m}$, and 3 to 12 septate. Based on morphology, the pathogen was identified as Pseudocercospora puerariicola Deighton (Guo and Hsieh 1995). Isolation was performed from the infected leaves to PDA using single-spore isolation method. After 26-day incubation at $28{ }^{\circ} \mathrm{C}, 2.8$ to $3.1 \mathrm{~cm}$ grayish brown colonies were observed. The ITS region was amplified using ITS1/ITS4 primers (White et al. 1990) and deposited in GenBank (MG601510). A BLAST search revealed 99\% identity with that of $P$. puerariicola isolate TNM3769 from Taiwan (KJ201939) (Kirschner and Liu 2014). A voucher specimen (JXAU-H2017188) was deposited in the Herbarium of College of Agronomy, Jiangxi Agricultural University. A conidial suspension $\left(1 \times 10^{5}\right.$ conidia $\left./ \mathrm{ml}\right)$ was sprayed on healthy plants incubated in a greenhouse at $28^{\circ} \mathrm{C}$, while control plants were sprayed with sterilized water. After 2 to 3 weeks, typical symptoms similar to those in the field appeared on the inoculated leaves, whereas the control leaves remained symptomless. P. puerariicola was successfully re-isolated from these diseased leaves. P. puerariicola was previously observed from some provinces in China, including Yunnan, Anhui, Zhejiang, Taiwan, Hunan, Guangdong, Guangxi (Tai 1979; Guo and Hsieh 1995). According to our knowledge, this is the first report of $P$. puerariicola causing leaf spot on kudzu in Jiangxi China.

Funding This study was supported by the National Natural Science Foundation of China (Grant No. 31460452).

\section{References}

Guo YL, Hsieh WH (1995) The genus Pseudocercospora in China. In: International academic publishers. Beijing, China

Kirschner R, Liu CL (2014) Mycosphaerellaceous fungi and new species of Venustosynnema and Zasmidium on ferns and fern allies in Taiwan. Phytotaxa 176:309-323

Tai FL (1979) Sylloge Fungorum Sinicorum. Sci. Press, Acad. Sin., Peking, 1527 pages

White TJ, Bruns T, Lee S, Taylor JW (1990) PCR protocols: a guide to methods and applications. Academic Press, San Diego

Jun Xi Jiang

jxau2011@126.com

1 College of Agronomy, Jiangxi Agricultural University,

Nanchang 330045, China 